\title{
Burhan Distribution with Structural Properties and Applications in Distinct Areas of Science
}

\author{
Aijaz Ahmad*, Muzamil Jallal, S. Quratul Ain and Rajnee Tripathi \\ Department of Mathematics, Bhagwant University, Ajmer, India \\ e-mail: ahmadaijaz4488@gmail.com ${ }^{*}$
}

\begin{abstract}
In this work a novel distribution has been explored referred as Burhan distribution. This distribution is obtained through convex combination of exponential and gamma distribution to analyse complex real-life data. The distinct structural properties of the formulated distribution have been derived and discussed. The behaviour of probability density function (pdf) and cumulative distribution function (cdf) are illustrated through different graphs. The estimation of the established distribution parameters are performed by maximum likelihood estimation method. Eventually the versatility of the established distribution is examined through two real life data sets.
\end{abstract}

\section{Introduction}

In biomedicine, engineering, economics, and other fields of science, statistical distribution plays essential role in modelling data. The exponential and gamma distributions are popular distributions for assessing statistical data and are regarded as life time distributions. Among these distributions, the exponential distribution has one parameter and several attractive statistical characteristics, such as memory less and a constant hazard rate. Various extensions of these distributions have been made in the statistical literature to lead greater flexibility. Lindley formulated a one parameter life time distribution with the following probability density function in (1958).

$$
f(y, \theta)=\frac{\theta^{2}}{\theta+1}(1+y) e^{-\theta y} ; \quad y>0, \theta>0
$$

Received: September 3, 2021; Accepted: September 25, 2021

2020 Mathematics Subject Classification: 60-XX, 62-XX, 11-Kxx.

Keywords and phrases: moments, moment generating function, reliability measures, mean deviations, maximum likelihood function. 
In recent years, researchers have worked extensively upon Lindley distribution, formulating one and two parameter distributions. For the modelling of diverse complex data, Ghitney et al. [2] conducted an in-depth study on the Lindley distribution, demonstrating that the Lindley distribution outranks the exponential distribution in terms of waiting times for bank customers. They also demonstrate that the contours of the hazard rate function of a Lindley distribution are increasing whereas the mean residual life is decreasing. Zakerzadeh and Dolati [3], Nadarajah et al. [4], they extended Lindley distribution with addition of new parameters and expounded the performance of the extended distribution through data sets. In recent years many authors have made different contributions to modify the Lindley distribution. Merovci [5], has introduced transmuted Lindley distribution and discussed its several properties. Sharma et al has introduced the inverse of the Lindley distribution and propounded its different properties. Shanker et al [6] developed a new life time distribution named Akash distribution which were proved superior then exponential and Lindley distribution. Sen et al. [7] has proposed a one parameter distribution called xgamma distribution and they have proved by an application that xgamma distribution provide better fit than exponential distribution. K.K Shukla [8], have suggested the Pranav distribution and studied its different properties. Aijaz et al. [9] has introduced transmuted inverse Lindley distributions and estimates its properties. Aijaz et al. [10] has developed a new distribution and name it Hamza distribution and studied its different properties. When it comes to analysing more complex data, these distributions have both advantages and limitations. In this study, an attempt is made to establish a new three parameter distribution that is considerably more pliable and produces better results than previous ones. The newly defined three parameter distribution's probability density function is as follows.

$$
f(y, \alpha, \beta, \theta)=\frac{\theta^{\beta+1}}{\alpha \theta^{\beta}+\Gamma(\beta)}\left(\alpha+\frac{y^{\beta}}{\beta}\right) e^{-\theta y} ; y>0, \alpha, \beta, \theta>0 .
$$

The suggested distribution is referred to as the Burhan distribution, and it has been developed by combining two distributions, exponential $(\theta)$ and gamma $(\beta, \theta)$, by employing the linear combination method.

$$
f(y, \alpha, \beta, \theta)=\rho g_{1}(y, \theta)+(1-\rho) g_{2}(y, \beta, \theta),
$$

Where

$$
\rho=\frac{\theta^{\beta+1}}{\alpha \theta^{\beta}+\Gamma(\beta)}
$$

$$
g_{1}(y, \theta)=\theta e^{-\theta y} ; y>0, \theta>0 ; \quad g_{2}(y, \theta)=\frac{y^{\beta}}{\Gamma(\beta+1)} e^{-\theta y} ; y>0, \beta, \theta>0 \text {. }
$$


Figures (1.1) and (1.2) expound few layouts of pdf of Burhan distribution for varying parameters.

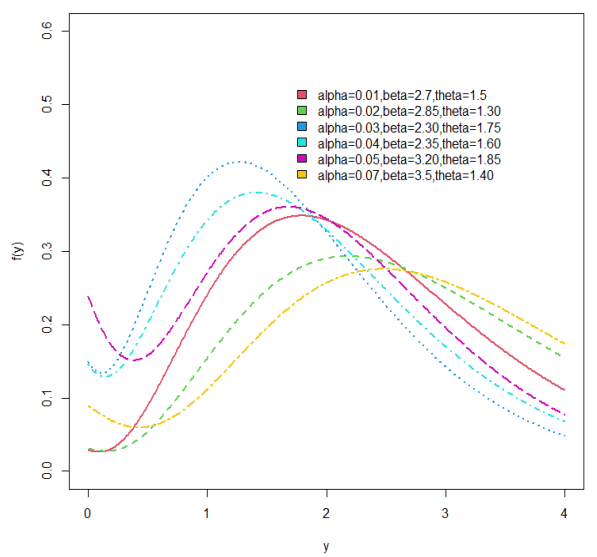

Figure 1.1: pdf of Burhan distribution under different values to parameters.

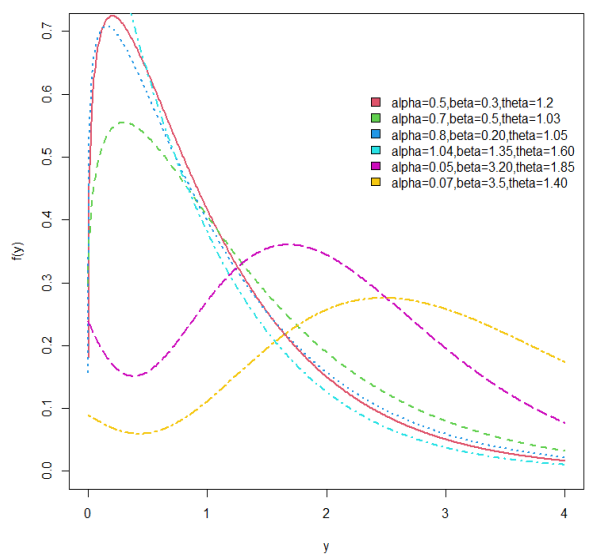

Figure 1.2: pdf of Burhan distribution under different values to parameters.

The associated cdf of equation (1.1) is given by

$$
F(y, \alpha, \beta, \theta)=1-\frac{\left(\alpha \beta \theta^{\beta} e^{-\theta y}+\Gamma(\beta+1, \theta y)\right)}{\beta\left(\alpha \theta^{\beta}+\Gamma(\beta)\right)} ; \quad y>0, \beta, \theta>0 .
$$

Figures (1.3) and (1.4) expounds few layouts of cdf of Burhan distribution for varying parameters

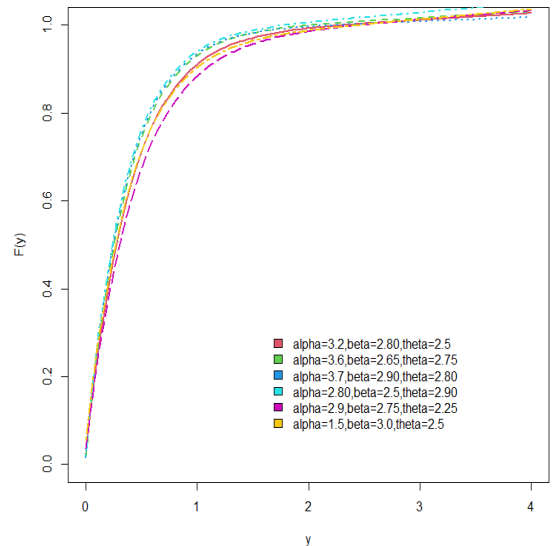

Figure 1.3: cdf of Burhan distribution under different values to parameters.

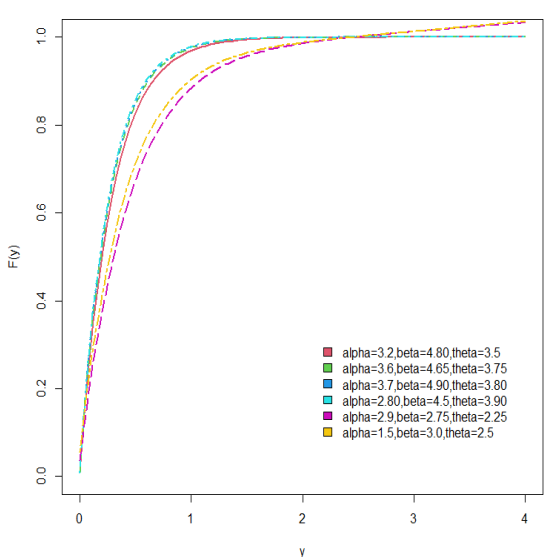

Figure 1.4: cdf of Burhan distribution under different values to parameters. 


\section{Mathematical Properties of Burhan Distribution}

\subsection{Moments of Burhan distribution}

Let $Y$ be a random variable follows Burhan distribution. Then $r^{\text {th }}$ moment denoted by $\mu_{r}^{\prime}$ is given as

$$
\begin{aligned}
\mu_{r}^{\prime}=E\left(Y^{r}\right) & =\int_{0}^{\infty} y^{r} f(y, \alpha, \beta, \theta) d y \\
& =\int_{0}^{\infty} y^{r} f(y, \alpha, \beta, \theta) d y \\
& =\int_{0}^{\infty} y^{r}\left(\alpha+\frac{y^{\beta}}{\beta}\right) e^{-\theta y} d y \\
& =\alpha \int_{0}^{\infty} y^{r} e^{-\theta y}+\frac{1}{\beta} \int_{0}^{\infty} y^{r+\beta} e^{-\theta y} d y
\end{aligned}
$$

After solving the integral, we have

$$
\mu_{r}^{\prime}=\frac{\alpha \beta \theta^{\beta} \Gamma(r+1)+\Gamma(r+\beta+1)}{\beta \theta^{r+\beta+1}} .
$$

Substituting $r=1,2,3,4$ we obtain first four moments about origin as

$$
\begin{gathered}
\mu_{1}^{\prime}=\frac{\alpha \theta^{\beta}+(\beta+1) \Gamma(\beta)}{\theta^{\beta+2}} \\
\mu_{2}^{\prime}=\frac{2 \alpha \theta^{\beta}+(\beta+2)(\beta+1) \Gamma(\beta)}{\theta^{\beta+3}} \\
\mu_{3}^{\prime}=\frac{6 \alpha \theta^{\beta}+(\beta+3)(\beta+2)(\beta+1) \Gamma(\beta)}{\theta^{\beta+4}} \\
\mu_{4}^{\prime}=\frac{24 \alpha \theta^{\beta}+(\beta+4)(\beta+3)(\beta+2)(\beta+1) \Gamma(\beta)}{\theta^{\beta+5}} .
\end{gathered}
$$

\subsection{Moment generating function of Burhan distribution}

Let $Y$ be a random variable follows Burhan distribution. Then the moment generating function of the distribution denoted by $M_{Y}(t)$ is given

$$
M_{Y}(t)=E\left(e^{t y}\right)=\int_{0}^{\infty} e^{t y} f(y, \alpha, \beta, \theta) d y
$$


Using Taylor's series

$$
\begin{aligned}
= & \int_{0}^{\infty}\left(1+t y+\frac{(t y)^{2}}{2 !}+\frac{(t y)^{3}}{3 !}+\ldots\right) f(y, \alpha, \beta, \theta) d y \\
= & \sum_{r=0}^{\infty} \frac{t^{r}}{r !} \int_{0}^{\infty} y^{r} f(y, \alpha, \beta, \theta) d y \\
= & \sum_{r=0}^{\infty} \frac{t^{r}}{r !} E\left(Y^{r}\right) \\
& M_{Y}(t)=\sum_{r=0}^{\infty} \frac{t^{r}}{r !} \frac{\alpha \beta \theta^{\beta} \Gamma(r+1)+\Gamma(r+\beta+1)}{\beta \theta^{r+\beta+1}} .
\end{aligned}
$$

\section{Renyi Entropy of Burhan Distribution}

If $Y$ denotes a continuous random variable having probability density function $f(y, \alpha, \beta, \theta)$. Then Renyi entropy is defined as

$$
T_{R}(\gamma)=\frac{1}{1-\gamma} \log \left\{\int_{0}^{\infty} f^{\gamma}(y) d y\right\},
$$

where $\gamma>0$ and $\gamma \neq 1$.

Thus, the Renyi entropy of Burhan distribution (1.1) is given as

$$
\begin{aligned}
T_{R}(\gamma) & =\frac{1}{1-\gamma} \log \left\{\frac{\theta^{(\beta+1) \gamma}}{\left(\alpha \theta^{\beta}+\Gamma(\beta)\right)^{\gamma}} \int_{0}^{\infty}\left(\alpha+\frac{y^{\beta}}{\beta}\right)^{\gamma} e^{-\gamma \theta y} d y\right\} \\
& =\frac{1}{1-\gamma} \log \left\{\frac{\theta^{(\beta+1) \gamma} \alpha^{\gamma}}{\left(\alpha \theta^{\beta}+\Gamma(\beta)\right)^{\gamma}} \int_{0}^{\infty}\left(1+\frac{y^{\beta}}{\beta}\right)^{\gamma} e^{-\gamma \theta y} d y\right\} .
\end{aligned}
$$

Using generalized binomial theorem, we have

$$
T_{R}(\gamma)=\frac{1}{1-\gamma} \log \left\{\sum_{r=0}^{\infty}\left(\begin{array}{l}
\gamma \\
r
\end{array}\right)\left(\frac{\alpha \theta^{(\beta+1)}}{\alpha \theta^{\beta}+\Gamma(\beta)}\right)^{\gamma} \frac{1}{(\alpha \beta)^{r}} \int_{0}^{\infty} y^{\beta r} e^{-\gamma \theta y} d y\right\} .
$$

After solving the integral, we get

$$
T_{R}(\gamma)=\frac{1}{1-\gamma} \log \left\{\sum_{r=0}^{\infty}\left(\begin{array}{l}
\gamma \\
r
\end{array}\right)\left(\frac{\alpha \theta^{(\beta+1)}}{\alpha \theta^{\beta}+\Gamma(\beta)}\right)^{\gamma} \frac{\Gamma(\beta r+1)}{(\alpha \beta)^{r}(\gamma \theta)^{\beta r+1}}\right\} .
$$




\section{Tsallis Entropy of Burhan Distribution}

Tsallis entropy of order $\gamma$ for Burhan distribution (1.1) is given as

where $\gamma>0$ and $\gamma \neq 1$

$$
S_{\gamma}=\frac{1}{\gamma-1}\left\{1-\int_{0}^{\infty} f^{\gamma}(y) d y\right\}
$$

$$
S_{\gamma}=\frac{1}{\gamma-1}\left\{1-\frac{\theta^{(\beta+1) \gamma}}{\left(\alpha \theta^{\beta}+\Gamma(\beta)\right)^{\gamma}} \int_{0}^{\infty}\left(\alpha+\frac{y^{\beta}}{\beta}\right)^{\gamma} e^{-\gamma \theta y} d y\right\} .
$$

After solving the integral, we get

$$
S_{\gamma}=\frac{1}{\gamma-1}\left(1-\sum_{r=0}^{\infty}\left(\begin{array}{l}
\gamma \\
r
\end{array}\right)\left(\frac{\alpha \theta^{(\beta+1)}}{\alpha \theta^{\beta}+\Gamma(\beta)}\right)^{\gamma} \frac{\Gamma(\beta r+1)}{(\alpha \beta)^{r}(\gamma \theta)^{\beta r+1}}\right) .
$$

\section{Mean Deviation from Mean of Burhan Distribution}

The quantity of scattering in a population is evidently measured to some extent by the totality of the deviations. Let $Y$ be a random variable from Burhan distribution with mean $\mu$. Then the mean deviation from mean is defined as

$$
\begin{aligned}
D(\mu) & =E(|Y-\mu|) \\
& =\int_{0}^{\infty}|Y-\mu| f(y) d y \\
& =\int_{0}^{\mu}(\mu-y) f(y) d y+\int_{\mu}^{\infty}(y-\mu) f(y) d y \\
& =\mu \int_{0}^{\mu} f(y) d y-\int_{0}^{\mu} y f(y) d y+\int_{\mu}^{\infty} y f(y) d y-\int_{\mu}^{\infty} \mu f(y) d y \\
& =\mu F(\mu)-\int_{0}^{\mu} y f(y) d y-\mu[1-F(\mu)]+\int_{\mu}^{\infty} y f(y) d y \\
& =2 \mu F(\mu)-2 \int_{0}^{\mu} y f(y) d y .
\end{aligned}
$$

Now

$$
\int_{0}^{\mu} y f(y) d y=\int_{0}^{\mu} y \frac{\theta^{\beta+1}}{\alpha \theta^{\beta}+\Gamma(\beta)}\left(\alpha+\frac{y^{\beta}}{\beta}\right) e^{-\theta y} d y
$$




$$
=\frac{\theta^{\beta+1}}{\alpha \theta^{\beta}+\Gamma(\beta)}\left\{\alpha \int_{0}^{\mu} y e^{-\theta y} d y+\frac{1}{\beta} \int_{0}^{\mu} y^{\beta+1} e^{-\theta y} d y\right\} .
$$

After solving the integral, we get

$$
\int_{0}^{\mu} y f(y) d y=\frac{\theta^{\beta+1}}{\alpha \theta^{\beta}+\Gamma(\beta)}\left\{\frac{\alpha}{\theta^{2}} \gamma(2, \theta \mu)+\frac{1}{\beta \theta^{\beta+2}} \gamma(\beta+2, \mu \theta)\right\} .
$$

Using equation (5.2) and (1.2) in equation (5.1), we obtain

$$
\begin{aligned}
D(\mu)= & 2 \mu-\frac{2 \mu\left(\alpha \beta \theta^{\beta} e^{-\theta \mu}+\Gamma(\beta+1, \theta \mu)\right)}{\beta\left(\alpha \theta^{\beta}+\Gamma(\beta)\right)} \\
& -\frac{2 \theta^{\beta+1}}{\alpha \theta^{\beta}+\Gamma(\beta)}\left\{\frac{\alpha}{\theta^{2}} \gamma(2, \theta \mu)+\frac{1}{\beta \theta^{\beta+2}} \gamma(\beta+2, \mu \theta)\right\} .
\end{aligned}
$$

\section{Mean Deviation from Median of Burhan Distribution}

Let $Y$ be a random variable from Burhan distribution with median $M$. Then the mean deviation from median is defined as

$$
\begin{aligned}
D(M) & =E(|Y-M|) \\
& =\int_{0}^{\infty}|Y-M| f(y) d y \\
& =\int_{0}^{M}(M-y) f(y) d y+\int_{M}^{\infty}(y-M) f(y) d y \\
& =M F(M)-\int_{0}^{M} y f(y) d y-M[1-F(M)]+\int_{M}^{\infty} y f(y) d y \\
& =\mu-2 \int_{0}^{M} y f(y) d y .
\end{aligned}
$$

Now

$$
\int_{0}^{M} y f(y) d y=\int_{0}^{M} y \frac{\theta^{\beta+1}}{\alpha \theta^{\beta}+\Gamma(\beta)}\left(\alpha+\frac{y^{\beta}}{\beta}\right) e^{-\theta y} d y .
$$


After solving the integral, we get

$$
\int_{0}^{M} y f(y) d y=\frac{\theta^{\beta+1}}{\alpha \theta^{\beta}+\Gamma(\beta)}\left\{\frac{\alpha}{\theta^{2}} \gamma(2, \theta M)+\frac{1}{\beta \theta^{\beta+2}} \gamma(\beta+2, M \theta)\right\} .
$$

Using equation (6.2) and (1.2) in equation (6.1), we obtain

$$
D(M)=\mu-\frac{2 \theta^{\beta+1}}{\alpha \theta^{\beta}+\Gamma(\beta)}\left\{\frac{\alpha}{\theta^{2}} \gamma(2, \theta M)+\frac{1}{\beta \theta^{\beta+2}} \gamma(\beta+2, M \theta)\right\} .
$$

\section{Reliability Measures}

Suppose $Y$ be a continuous random variable with $\operatorname{cdf} F(y), y \geq 0$. Then its reliability function which is also called survival function is defined as

$$
S(y)=p_{r}(Y>y)=\int_{y}^{\infty} f(y) d y=1-F(y) .
$$

Therefore, the survival function for Burhan distribution is given as

$$
\begin{aligned}
S(y, \alpha, \beta, \theta) & =1-F(y, \alpha, \beta, \theta) \\
& =\frac{\left(\alpha \beta \theta^{\beta} e^{-\theta y}+\Gamma(\beta+1, \theta y)\right)}{\beta\left(\alpha \theta^{\beta}+\Gamma(\beta)\right)} .
\end{aligned}
$$

The hazard rate function of a random variable $y$ is given as

$$
h(y, \alpha, \beta, \theta)=\frac{f(y, \alpha, \beta, \theta)}{S(y, \alpha, \beta, \theta)} .
$$

Using equation (1.1) and equation (7.1) in (7.2), we get

$$
h(y, \alpha, \beta, \theta)=\frac{\beta \theta^{\beta+1}\left(\alpha+\frac{y^{\beta}}{\beta}\right) e^{-\theta y}}{\left(\alpha \beta \theta^{\beta} e^{-\theta y}+\Gamma(\beta+1, \theta y)\right)} .
$$

Figure (7.1) and (7.2) expound few layouts of hazard rate function of Burhan distribution for varying parameters. 


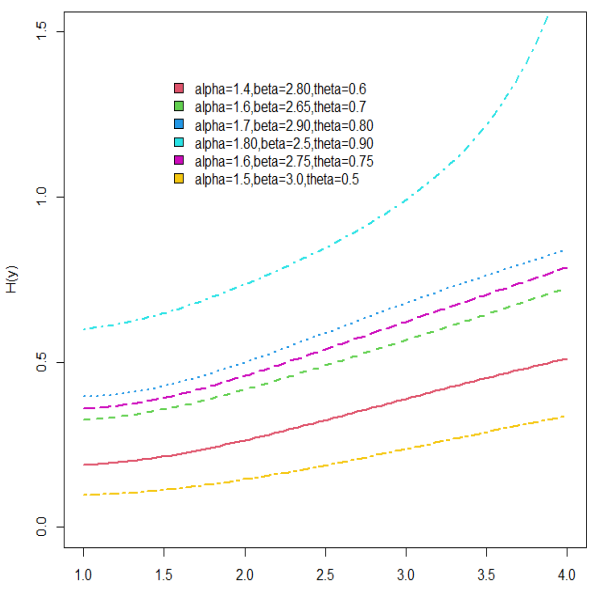

Figure 7.1: HRF of Burhan distribution under different values to parameters.

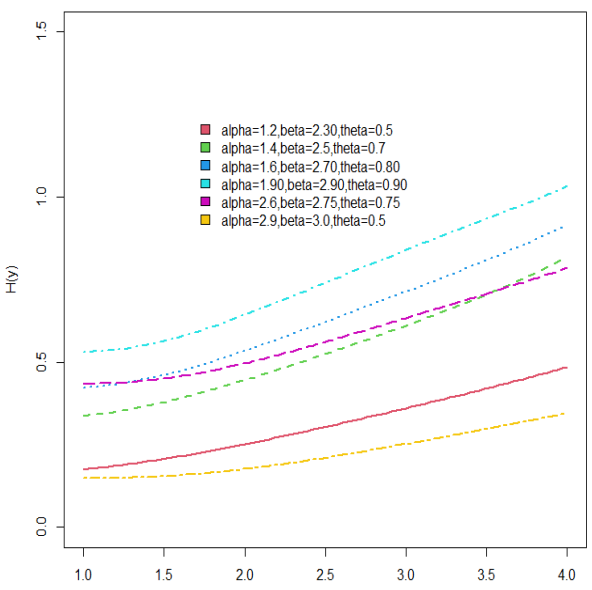

Figure 7.2: HRF of Burhan distribution under different values to parameters.

The cumulative hazard rate function is given as

$$
H(y, \alpha, \beta, \theta)=-\log [F(y, \alpha, \beta, \theta)] .
$$

Using equation (1.2), we get

$$
H(y, \alpha, \beta, \theta)=-\log \left[1-\frac{\left(\alpha \beta \theta^{\beta} e^{-\theta y}+\Gamma(\beta+1, \theta y)\right)}{\beta\left(\alpha \theta^{\beta}+\Gamma(\beta)\right)}\right] .
$$

Mean residual function of random variable $y$ can be obtained as

$$
\begin{aligned}
m(y, \alpha, \beta, \theta) & =\frac{1}{S(y, \alpha, \beta, \theta)} \int_{y}^{\infty} t f(t, \alpha, \beta, \theta) d t-y \\
& =\frac{\beta\left(\alpha \theta^{\beta}+\Gamma(\beta)\right)}{\alpha \beta \theta^{\beta} e^{-\theta y}+\Gamma(\beta+1, \theta y)} \int_{0}^{\infty} \frac{\theta^{\beta+1}}{\alpha \theta^{\beta}+\Gamma(\beta)} t\left(\alpha+\frac{t^{\beta}}{\beta}\right) e^{-\theta t} d t-y \\
& =\frac{\beta \theta^{\beta+1}}{\alpha \beta \theta^{\beta} e^{-\theta y}+\Gamma(\beta+1, \theta y)}\left\{\int_{0}^{\infty} t e^{-\theta t} d t+\frac{1}{\beta} \int_{0}^{\infty} t^{\beta+1} e^{-\theta t} d t\right\}-y .
\end{aligned}
$$

After solving the integral, we get

$$
m(y, \alpha, \beta, \theta)=\frac{\alpha \beta \theta^{\beta}(\theta y+1) e^{-\theta y}+\Gamma(\beta+2, \theta y)}{\theta\left(\alpha \beta \theta^{\beta} e^{-\theta y}+\Gamma(\beta+1, \theta y)\right)}-y .
$$




\section{Order Statistics of Burhan Distribution}

Let $Y_{(1)}, Y_{(2)}, Y_{(3)}, \ldots, Y_{(n)}$ denotes the order statistics of a random sample drawn from a continuous distribution with $\operatorname{cdf} F(y)$ and pdf $f(y)$, then the pdf of $Y_{(k)}$ is given by

$$
f_{Y(k)}(Y, \theta)=\frac{n !}{(k-1) !(n-k) !} f_{Y}(y)\left[F_{Y}(y)\right]^{k-1}\left[1-F_{Y}(y)\right]^{n-k} \quad k=1,2,3 \ldots, n .
$$

Substitute the equation (1.1) and (2.2) in equation (8.1), we obtain the probability function of $k^{\text {th }}$ order statistics of Burhan distribution is given by

$$
\begin{aligned}
& f_{Y(k)}(y, \theta)=\frac{n !}{(k-1) !(n-k) !} \frac{\theta^{\beta+1}}{\alpha \theta^{\beta}+\Gamma(\beta)}\left(\alpha+\frac{y^{\beta}}{\beta}\right) e^{-\theta y}\left[1-\frac{\left(\alpha \beta \theta^{\beta} e^{-\theta y}+\Gamma(\beta+1, \theta y)\right)}{\beta\left(\alpha \theta^{\beta}+\Gamma(\beta)\right)}\right]^{k-1} \\
& {\left[\frac{\left(\alpha \beta \theta^{\beta} e^{-\theta y}+\Gamma(\beta+1, \theta y)\right)}{\beta\left(\alpha \theta^{\beta}+\Gamma(\beta)\right)}\right]^{n-k} \cdot }
\end{aligned}
$$

Then, the pdf of first order statistics $Y_{(1)}$ of Burhan distribution given by

$$
f_{Y(1)}(y, \theta)=\frac{n \theta^{\beta+1}}{\alpha \theta^{\beta}+\Gamma(\beta)}\left(\alpha+\frac{y^{\beta}}{\beta}\right) e^{-\theta y}\left[\frac{\left(\alpha \beta \theta^{\beta} e^{-\theta y}+\Gamma(\beta+1, \theta y)\right)}{\beta\left(\alpha \theta^{\beta}+\Gamma(\beta)\right)}\right]^{1-k} .
$$

And the pdf of $n$th order statistics $Y_{(n)}$ of Burhan distribution is given by

$$
f_{Y(n)}(y, \theta)=\frac{n \theta^{\beta+1}}{\alpha \theta^{\beta}+\Gamma(\beta)}\left(\alpha+\frac{y^{\beta}}{\beta}\right) e^{-\theta y}\left[1-\frac{\left(\alpha \beta \theta^{\beta} e^{-\theta y}+\Gamma(\beta+1, \theta y)\right)}{\beta\left(\alpha \theta^{\beta}+\Gamma(\beta)\right)}\right]^{1-k} .
$$

\section{Maximum Likelihood Estimator of Burhan Distribution}

Let $Y_{1}, Y_{2}, \ldots, Y_{n}$ be a random sample of size $n$ from Burhan distribution. Then its likelihood function is given by

$$
\begin{aligned}
l & =\prod_{i=1}^{n} f\left(y_{i}, \alpha, \beta, \theta\right) \\
& =\left(\frac{\theta^{\beta+1}}{\alpha \theta^{\beta}+\Gamma(\beta)}\right)^{n} \prod_{i=1}^{n}\left(\alpha+\frac{y_{i}}{\beta}\right) e^{-\theta \sum_{i=1}^{n} y_{i}} .
\end{aligned}
$$


The log likelihood function is given as

$$
\log l=n(\beta+1) \log \theta-n \log \left(\alpha \theta^{\beta}+\Gamma(\beta)\right)+\sum_{i=1}^{n} \log \left(\alpha+\frac{y_{i}^{\beta}}{\beta}\right)-\theta \sum_{i=1}^{n} y_{i} .
$$

The partial derivatives of equation (9.1), with respect parameters are given as

$$
\begin{gathered}
\frac{\partial \log l}{\partial \alpha}=\frac{-n \theta^{\beta}}{\alpha \theta^{\beta}+\Gamma(\beta)}+\sum_{i=1}^{n} \frac{\beta}{\alpha \beta+y_{i}^{\beta}} \\
\frac{\partial \log l}{\partial \beta}=n \log \theta-\frac{n\left(\alpha \theta^{\beta} \log \theta+\Gamma^{\prime}(\beta)\right)}{\alpha \theta^{\beta}+\Gamma(\beta)}+\sum_{i=1}^{n} \frac{\left(\beta \log y_{i}-1\right) y_{i}^{\beta}}{\beta\left(\alpha \beta+y_{i}^{\beta}\right)} \\
\frac{\partial \log l}{\partial \theta}=\frac{n(\beta+1)}{\theta}-\frac{n \alpha \beta \theta^{\beta-1}}{\alpha \theta^{\beta}+\Gamma(\beta)}-\sum_{i=1}^{n} y_{i} .
\end{gathered}
$$

From equations (9.2), (9.3) and (9.4), we have obtained a system of non-linear equations which cannot be expressed in compact form and is difficult to solve explicitly for $\alpha, \beta$ and $\theta$. Applying the iterative methods such as Newton-Raphson method, secant method, Regula-falsi method etc. The MLE of theparameters denoted as $\hat{\zeta}(\hat{\alpha}, \hat{\beta}, \hat{\theta})$ of $\zeta(\alpha, \beta, \theta)$ can be obtained by using the above methods.

For interval estimation and hypothesis tests on the model parameters, an information matrix is required. The 3 by 3 observed matrix is

$$
I(\zeta)=-\frac{1}{n}\left[\begin{array}{lll}
E\left(\frac{\partial^{2} \log l}{\partial \alpha^{2}}\right) & E\left(\frac{\partial^{2} \log l}{\partial \alpha \partial \beta}\right) & E\left(\frac{\partial^{2} \log l}{\partial \alpha \partial \theta}\right) \\
E\left(\frac{\partial^{2} \log l}{\partial \beta \partial \alpha}\right) & E\left(\frac{\partial^{2} \log l}{\partial \beta^{2}}\right) & E\left(\frac{\partial^{2} \log l}{\partial \beta \partial \theta}\right) \\
E\left(\frac{\partial^{2} \log l}{\partial \theta \partial \alpha}\right) & E\left(\frac{\partial^{2} \log l}{\partial \theta \partial \beta}\right) & E\left(\frac{\partial^{2} \log l}{\partial \theta^{2}}\right)
\end{array}\right]
$$

The elements of above information matrix can obtain by differentiating equations (9.2), (9.3) and (9.4) again partially. Under standard regularity conditions when $n \rightarrow \infty$ the distribution of $\hat{\zeta}$ can be approximated by a multivariate normal $N\left(0, I(\hat{\zeta})^{-1}\right)$ distribution to construct approximate confidence interval for the parameters.

Hence the approximate $100(1-\psi) \%$ confidence interval for $\alpha, \beta$ and $\theta$ are respectively given by 


$$
\hat{\alpha} \pm Z_{\frac{\psi}{2}} \sqrt{I_{\alpha \alpha}^{-1}(\hat{\zeta})}, \quad \hat{\beta} \pm Z_{\frac{\psi}{2}} \sqrt{I_{\beta \beta}^{-1}(\hat{\zeta})} \text { and } \quad \hat{\theta} \pm Z_{\frac{\psi}{2}} \sqrt{I_{\theta \theta}^{-1}(\hat{\zeta})}
$$

where $Z_{\frac{\psi}{2}}$ denotes the $\zeta^{\text {th }}$ percentile of the standard normal distribution.

\section{Applications}

This section exhibits the adaptability of the formulated distribution by applying realworld data sets. The suggested distribution is compared to the Shanker distribution (SHD), the Akash distribution (AD), the Ishita distribution (ID), the Pranav distribution (PD), the Rani distribution (RD), the Prakaamy distribution (PKD), and the Lindley distribution. We were efficient in outranking the specified distribution.

In order to compare the above distribution models, we consider the criteria like AIC (Akaike information criterion), CAIC (corrected Akaike information criterion), BIC (Bayesian information criterion). Among the above distributions, the better distribution is considered having lesser values of AIC, CAIC, HQIC and BIC.

Data set 1: This data is obtained from Murthy et al. [13], which represents failure times of 84 Aircraft Windshield. The data follows:

$0.040,1.866,2.385,3.443,0.301,1.876,2.481,3.467,0.309,1.899,2.610,3.478,0.557$, $1.911,2.625,3.578,0.943,1.912,2.632,3.595,1.070,1.914,2.646,3.699,1.124,1.981$, $2.661,3.779,1.248,2.010,2.688,3.924,1.281,2.038,2.82,3,4.035,1.281,2.085,2.890$, $4.121,1.303,2.089,2.902,4.167,1.432,2.097,2.934,4.240,1.480,2.135,2.962,4.255$, $1.505,2.154,2.964,4.278,1.506,2.190,3.000,4.305,1.568,2.194,3.103,4.376,1.615$, $2.223,3.114,4.449,1.619,2.224,3.117,4.485,1.652,2.229,3.166,4.570,1.652,2.300$, $3.344,4.602,1.757,2.324,3.376,4.663$.

Table 10.1: Descriptive statistics of data $1^{\text {st }}$.

\begin{tabular}{|l|c|c|c|c|c|c|c|}
\hline Min & $Q_{1}$ & Median & Mean & $Q_{3}$ & Skew. & Kurt. & Max \\
\hline 0.040 & 1.866 & 2.385 & 2.563 & 3.376 & 0.086 & 2.365 & 4.663 \\
\hline
\end{tabular}


Table 10.2: The ML estimates of the unknown parameters for data set $1^{\text {st }}$.

\begin{tabular}{|c|c|c|c|c|c|c|}
\hline \multirow[t]{2}{*}{ Model } & \multirow[t]{2}{*}{$\hat{\alpha}$} & \multirow[t]{2}{*}{$\hat{\beta}$} & \multirow[t]{2}{*}{$\hat{\theta}$} & \multicolumn{3}{|c|}{ S.E } \\
\hline & & & & $\hat{\alpha}$ & $\hat{\beta}$ & $\hat{\theta}$ \\
\hline BHD & 0.0206 & 5.6641 & 2.4893 & 0.0129 & 1.0566 & 0.4044 \\
\hline SHD & ...... & ...... & 0.6440 & ...... & ........ & 0.0459 \\
\hline $\mathrm{AD}$ & ...... & ...... & 0.9337 & ...... & ....... & 0.056 \\
\hline ID & ....... & ...... & 0.9181 & ....... & ....... & 0.0495 \\
\hline PD & ...... & ...... & 1.2104 & ...... & ...... & 0.0530 \\
\hline $\mathrm{RD}$ & ...... & ...... & 1.5155 & ...... & ...... & 0.0563 \\
\hline PKD & ...... & ...... & 1.7287 & ...... & ...... & 0.0710 \\
\hline LD & ...... & ...... & 0.6296 & ...... & ...... & 0.0502 \\
\hline
\end{tabular}

Table 10.3: Performance of distributions for data set $1^{\text {st }}$.

\begin{tabular}{|c|l|l|l|l|l|}
\hline Model & $-\log l$ & AIC & CAIC & BIC & HQIC \\
\hline BHD & 127.63 & 261.26 & 261.55 & 268.59 & 264.21 \\
\hline SHD & 151.55 & 305.10 & 305.14 & 307.54 & 306.08 \\
\hline AD & 147.46 & 296.93 & 296.97 & 299.37 & 297.91 \\
\hline ID & 146.83 & 295.66 & 295.71 & 298.10 & 296.64 \\
\hline PD & 144.40 & 290.81 & 290.85 & 293.25 & 291.79 \\
\hline RD & 143.12 & 288.24 & 288.29 & 290.68 & 289.22 \\
\hline PKD & 188.70 & 379.40 & 379.44 & 381.84 & 380.38 \\
\hline LD & 153.96 & 309.93 & 309.98 & 312.37 & 310.91 \\
\hline
\end{tabular}

Data set 2: The data set represents the survival times (in days) of 72 guinea pigs infected with virulent tubercle bacilli, observed and reported by Bjerkedal [12]. The data are as follows: 
$0.1,0.33,0.44,0.56,0.59,0.59,0.72,0.74,0.92,0.93,0.96,1,1,1.02,1.05,1.07,1.07$, $1.08,1.08,1.08,1.09,1.12,1.13,1.15,1.16,1.2,1.21,1.22,1.22,1.24,1.3,1.34,1.36$, $1.39,1.44,1.46,1.53,1.59,1.6,1.63,1.68,1.71,1.72,1.76,1.83,1.95,1.96,1.97,2.02$, $2.13,2.15,2.16,2.22,2.3,2.31,2.4,2.45,2.51,2.53,2.54,2.78,2.93,3.27,3.42,3.47$, $3.61,4.02,4.32,4.58,5.55,2.54,0.77$.

Table 10.4: Descriptive Statistics of data $2^{\text {nd }}$

\begin{tabular}{|l|l|l|l|l|l|l|l|}
\hline Min & $\mathrm{Q}_{1}$ & Median & Mean & $\mathrm{Q}_{3}$ & Skew. & Kurt. & Max \\
\hline 0.100 & 1.077 & 1.450 & 1.754 & 2.240 & 1.328 & 4.913 & 5.550 \\
\hline
\end{tabular}

Table 10.5: The ML estimates of the unknown parameters for data set $2^{\text {nd }}$.

\begin{tabular}{|c|c|c|c|c|c|c|}
\hline \multirow[t]{2}{*}{ Model } & \multirow[t]{2}{*}{$\hat{\alpha}$} & \multirow[t]{2}{*}{$\hat{\beta}$} & \multirow[t]{2}{*}{$\hat{\theta}$} & \multicolumn{3}{|c|}{ S.E } \\
\hline & & & & $\hat{\alpha}$ & $\hat{\beta}$ & $\hat{\theta}$ \\
\hline BHD & 0.0067 & 2.4143 & 1.9124 & 0.0085 & 0.6517 & 0.3729 \\
\hline SHD & ...... & ...... & 0.8667 & ...... & ....... & 0.0675 \\
\hline $\mathrm{AD}$ & ...... & ...... & 1.2227 & ...... & ........ & 0.0815 \\
\hline ID & ...... & ...... & 1.1664 & ...... & ...... & 0.0680 \\
\hline PD & ...... & $\ldots \ldots$ & 1.4780 & $\ldots \ldots$ & $\ldots \ldots$ & 0.068 \\
\hline $\mathrm{RD}$ & ...... & ...... & 1.7994 & $\ldots \ldots$ & ...... & 0.0699 \\
\hline PKD & ...... & ...... & 2.1118 & ...... & ...... & 0.0853 \\
\hline LD & ...... & ...... & 0.8744 & ...... & ...... & 0.0771 \\
\hline
\end{tabular}

Table 10.6: Performance of distributions for data set $2^{\text {nd }}$.

\begin{tabular}{|c|l|l|l|l|l|}
\hline Model & $-\log l$ & AIC & CAIC & BIC & HQIC \\
\hline BHD & 94.06 & 194.12 & 194.48 & 200.95 & 196.84 \\
\hline SHD & 105.98 & 213.97 & 214.02 & 214.87 & 216.24 \\
\hline AD & 107.06 & 216.13 & 216.19 & 218.41 & 217.04 \\
\hline ID & 108.03 & 218.06 & 218.12 & 220.34 & 218.97 \\
\hline PD & 112.53 & 227.07 & 227.12 & 229.34 & 227.97 \\
\hline RD & 118.26 & 238.53 & 238.58 & 240.80 & 239.43 \\
\hline PKD & 176.87 & 355.75 & 355.81 & 358.03 & 356.66 \\
\hline LD & 106.52 & 215.05 & 215.10 & 217.32 & 215.95 \\
\hline
\end{tabular}




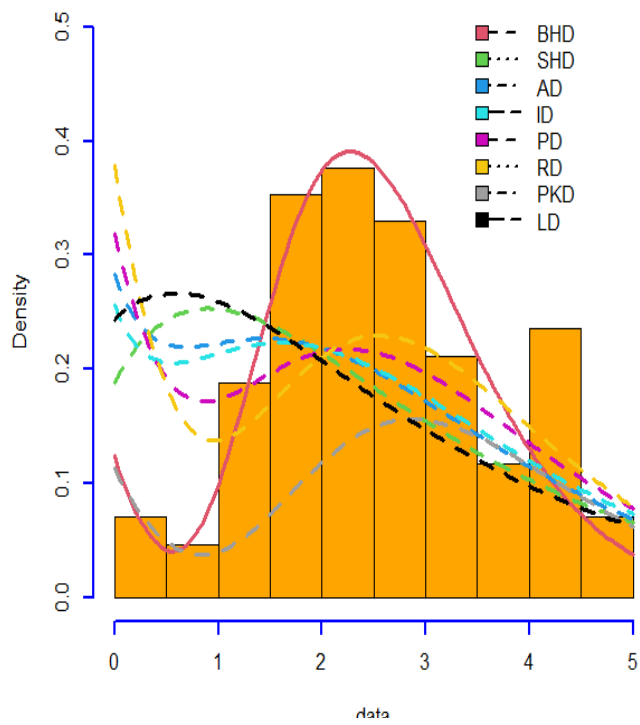

Figure 10.1: Fitted pdf's for data set I.

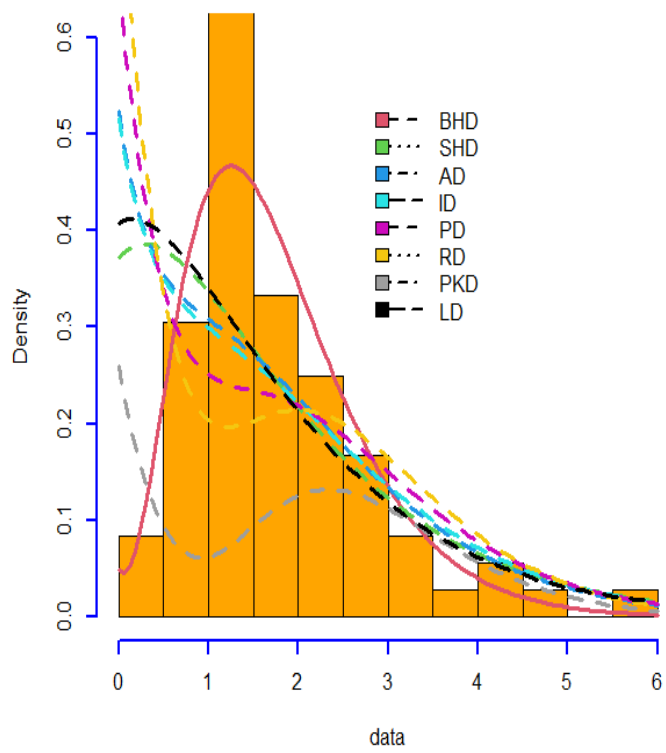

Figure 10.2: Fitted pdf's for data set II.

It is clear from Table 10.3 and 10.6 that Burhan distribution (BHD) has least values of - $\log$, AIC, CAIC, BIC and HQIC when compared with competitive distributions. We accomplish that Burhan distribution provides an adequate fit than compared distributions. 


\section{Conclusion}

In the present paper a novel distribution has been formulated by employing convex combination of exponential and gamma distribution stated as Burhan distribution (BHD). Its several properties including moments, moment generating function, survival function, hazard rate function, mean residual life function, mean deviations, order statistics, Renyi entropy and Tsallis entropy have been discussed. The parameters of the distribution have been estimated by known method of maximum likelihood estimator. Finally the performance of the model has been examined through two data sets and compared which shows that Burhan distribution gives an adequate fit for the data sets.

\section{References}

[1] D. V. Lindley, Fiducial distributions and Bayes' theorem, Journal of Royal Statistical Society Series B 20 (1958), 102-107. https://doi.org/10.1111/j.2517-6161.1958.tb00278.x

[2] M.E. Ghitany, B. Atieh and S. Nadarajah, Lindley distribution and its application, Mathematics and Computers in Simulation 78 (2008), 493-506.

https://doi.org/10.1016/j.matcom.2007.06.007

[3] H. Zakerzadeh and A. Dolati, Generalized Lindley distribution, Journal of Mathematical Extension 3(2) (2009), 13-25.

[4] S. Nadarajah, H. S. Bakouch and R. Tahmasbi, A generalized Lindley distribution, Sankhya B 73 (2011), 331-359. https://doi.org/10.1007/s13571-011-0025-9

[5] F. Merovci, Transmuted Lindley distribution, Int. J. Open Problems Compt. Math. 6 (2013), 63-72. https://doi.org/10.12816/0006170

[6] R. Shanker, Akash distribution and its applications, International Journal of Probability and Statistics 4(3) (2015), 65-75.

[7] S. Sen, S. Maiti and N. Chandra, The xgamma distribution: Statistical properties and application, Journal of Modern Applied Statistical Methods 15(1) (2016), 774-788. https://doi.org/10.22237/jmasm/1462077420

[8] K.K. Shukla, Pranav distribution with properties and its applications, Biom. Biostat. Int. J. 7(3) (2018), 244-254. https://doi.org/10.15406/bbij.2018.07.00215

[9] A. Aijaz, A Ahmad and R. Tripathi, Transmuted inverse Lindley distribution: Statistical properties and applications, Science, Technology and Development 9 (2020), 1-10.

[10] A. Aijaz, M. Jallal, S. Qurat Ul Ain and R. Tripathi, The Hamza distribution with 
statistical properties and applications, Asian Journal of Probability and Statistics 8(1) (2020), 28-42. https://doi.org/10.9734/ajpas/2020/v8i130198

[11] A. Aijaz, A Ahmad and R. Tripathi, Sauleh distribution with statistical properties and applications, Annal Biostat. and Biomed Appli. 4(1) (2020), 1-5.

[12] T. Bjerkedal, Acquisition of resistance in guinea pigs infected with different doses of virulent tubercle bacilli, American Journal of Epidemiology 72(1) (1960), 130-148. https://doi.org/10.1093/oxfordjournals.aje.a120129

[13] D.N.P. Murthy, M. Xie and R. Jiang, Weibull Models, Wiley, 2004.

This is an open access article distributed under the terms of the Creative Commons Attribution License (http://creativecommons.org/licenses/by/4.0/), which permits unrestricted, use, distribution and reproduction in any medium, or format for any purpose, even commercially provided the work is properly cited. 